\title{
Analysis of the Cob(II)alamin - 5'-Deoxy-3',4'-Anhydroadenosyl Radical Triplet Spin System in the Active Site of Diol Dehydrase
}

\author{
Steven O. Mansoorabadi $\ddagger$, Olafur Th. Magnusson§, Russell R. Poyner, Perry A. Frey, and \\ George H. Reed \\ Department of Biochemistry, University of Wisconsin-Madison, Madison, Wisconsin 53726-4087
}

\section{Abstract}

A triplet spin system $(S=1)$ is detected by low-temperature electron paramagnetic resonance (EPR) spectroscopy in samples of diol dehydrase and the functional adenosylcobalamin (AdoCbl) analog $5^{\prime}$-deoxy-3',4'-anhydroadenosylcobalamin (anAdoCbl). Different spectra are observed in the presence and absence of the substrate $(R, S)$-1,2-propanediol. In both cases, the spectra include a prominent half-field transition $\left(\Delta \mathrm{M}_{\mathrm{S}}=2\right)$ that is a hallmark of strongly-coupled triplet spin systems. The appearance of ${ }^{59} \mathrm{Co}$-hyperfine splitting in the EPR signals as well as the positions ( $g$-values) of the signals in the spectra show that half of the triplet spin is contributed by the low spin $\mathrm{Co}^{2+}$ of $\mathrm{cob}$ (II)alamin. Linewidth effects from isotopic labeling $\left({ }^{13} \mathrm{C}\right.$ and $\left.{ }^{2} \mathrm{H}\right)$ in the $5^{\prime}$-deoxy-3', $4^{\prime}$ -

anhydroribosyl ring demonstrate that the other half of the spin triplet is from an allylic 5'-deoxy-3', 4 '-anhydroadenosyl (anhydroadenosyl) radical. The zero-field splitting (ZFS) tensors describing the magnetic dipole-dipole interactions of the component spins of the triplets have rhombic symmetry because of electron spin delocalization within the organic radical component and nearness of the radical to the low spin $\mathrm{Co}^{2+}$. The dipole-dipole interaction was modeled as a summation of pointdipole interactions involving the spin-bearing orbitals of the anhydroadenosyl radical and cob(II) alamin. Geometries which are consistent with the ZFS tensors in the presence and absence of substrate position the $5^{\prime}$-carbon of the anhydroadenosyl radical $3.5 \AA$ and $4.1 \AA$ and from $\mathrm{Co}^{2+}$, respectively. Homolytic cleavage of the cobalt-carbon bond of the analog in the absence of substrate indicates that, in diol dehydrase, binding of the coenzyme to the protein weakens the bond prior to binding of substrate.

\begin{abstract}
A common characteristic of AdoCbl ${ }^{1}$-dependent enzymes is the generation and utilization of enzyme-bound organic radicals (1-3). The $\mathrm{Co}-\mathrm{C}^{\prime}$ bond of AdoCbl is relatively weak having a bond dissociation energy of $\sim 30 \mathrm{kcal} \mathrm{mol}^{-1}$ (4). A prevalent mechanism for AdoCbldependent enzymes begins with the homolytic cleavage of the $\mathrm{Co}-\mathrm{C} 5^{\prime}$ bond to give $\mathrm{cob}$ (II) alamin and the 5'-deoxyadenosyl radical (1). The 5'-deoxyadenosyl radical being an unstabilized, primary alkyl radical is expected to have a short lifetime, low concentration, and a high reactivity towards $\mathrm{H}$ atom abstraction. Thus far, these properties of the 5'-deoxyadenosyl radical have precluded direct observation of the radical by EPR spectroscopy. Cleavage of the
\end{abstract}

\footnotetext{
*Address Correspondence to: George H. Reed, University of Wisconsin, 1710 University Avenue, Madison, WI 53726-4087, Telephone: (608) 262-0509, FAX: (608) 265-2904, Email: reed@biochem.wisc.edu.

Fresent address: University of Texas College of Pharmacy, Dept. Med. Chem., Austin, TX 78712

\$Present address: deCode Genetics/Drug Discovery, Sturlugata 8, IS - 101 Reykjavik, Iceland

${ }^{\dagger}$ This research was supported by NIH grants GM35752 (G.H.R.) and DK28607 (P.A.F). S.O.M. was supported in part by NIH Predoctoral Training Grant T32 GM08293 in Molecular Biophysics.

${ }^{1}$ Abbreviations: AdoCbl, 5'-deoxyadenosylcobalamin; anAdoCbl, 5'-deoxy-3',4'-anhydroadenosylcobalamin; anATP, 3',4'anhydroadenosine triphosphate; anhydroadenosyl radical, 5'-deoxy-3',4'-anhydroadenosyl radical; BDE, bond dissociation energy; DMB, dimethylbenzimidazole; DDH, diol dehydrase; EPR, electron paramagnetic resonance; ENDOR, electron nuclear double resonance; SAM, $S$-adenosyl-L-methionine; ZFS, zero-field splitting; RMSD, root-mean-square deviation.
} 
$\mathrm{Co}-\mathrm{C} 5^{\prime}$ bond is kinetically coupled to $\mathrm{H}$ atom abstraction from a substrate molecule in the reactions of methylmalonyl-CoA mutase (5), glutamate mutase (6), ethanolamine ammonialyase (7), and diol dehydrase (8). These kinetic experiments have highlighted the transient nature of the 5'-deoxyadenosyl radical species. In the case of the AdoCbl-dependent Class II ribonucleoside triphosphate reductase the 5'-deoxyadenosyl radical abstracts an $\mathrm{H}$ atom from the thiol of Cys 408 of the protein (9). An allosteric effector-dependent epimerization of chiral $\left[5^{\prime}-{ }^{2} \mathrm{H}_{1}\right] \mathrm{AdoCbl}$ by the $\mathrm{C} 408 \mathrm{~A}$ and $\mathrm{C} 408 \mathrm{~S}$ mutant forms of the enzyme requires the transient existence of the 5'-deoxyadenosyl radical (10).

DDH (diol dehydrase, EC:4.2.1.28) uses 2- and 3-carbon vicinal diols as substrates and catalyzes their transformation into aldehydes $(8,11)$. The minimal mechanism for DDH with 1,2-propanediol as the substrate is depicted in Scheme 1. Reversible cleavage of the Co-C5' bond of AdoCbl generates the 5'-deoxyadenosyl radical a, which abstracts a hydrogen atom from $\mathbf{C} 1$ of 1,2-propanediol to produce 5'-deoxyadenosine $\mathbf{b}$ and the substrate radical $\mathbf{c}$. The substrate radical undergoes isomerization to the product-related radical $\mathbf{d}$ by an unknown mechanism. Hypothetical mechanisms for the radical rearrangement include the formation of a radical cation (12) or a radical anion intermediate (13). Concerted mechanisms having transition state stabilization by partial proton transfer or deprotonation of the spectator $-\mathrm{OH}$ group have also been discussed $(14,15)$. Crystal structures of DDH reveal that the vicinyl $-\mathrm{OH}$ groups of 1,2-propanediol are coordinated to $\mathrm{K}^{+}$, and $\mathrm{K}^{+}$may be involved in catalysis (16, 17). Magnetic interactions between active site radicals and the nuclear spins of ${ }^{39} \mathrm{~K}$ and $203,205 \mathrm{Tl}$ have, however, escaped detection by EPR and ENDOR (18). Moreover, site directed mutagenesis has revealed essential roles of Glu- $\alpha 170$ and Asp- $\alpha 335$ (19) in putative $\mathrm{H}$-bonding contacts with the vicinal - $\mathrm{OH}$ groups of the substrate. Hydrogen transfer from 5'deoxyadenosine $\mathbf{b}$ to the radical $\mathbf{d}$ regenerates the 5 '-deoxyadenosyl radical, which recombines with cob(II)alamin. The enzyme catalyzes the dehydration of the gem-diol to complete the reaction (20). Reactions of the DDH-AdoCbl complex with substrates or inhibitors elicit EPR signals corresponding to organic radicals spin coupled to the low spin $\mathrm{Co}^{2+}$ of cob(II)alamin $(18,21-26)$.

AnAdoCbl, shown in Scheme 2, is an analog of AdoCbl. AnAdoCbl has a Co-C5' bond dissociation energy which is $\sim 6 \mathrm{kcal} \mathrm{mol}^{-1}$ weaker than the corresponding bond in AdoCbl (27). The weakened $\mathrm{Co}-\mathrm{C} 5^{\prime}$ bond in anAdoCbl is attributed to allylic stabilization in the anhydroadenosyl radical relative to the 5'-deoxyadenosyl radical. Structures of the two radicals are compared in Scheme 3. AnAdoCbl is a functional coenzyme for $\mathrm{DDH}$, displaying a turnover rate $0.02 \%$ of that with AdoCbl as coenzyme (28). Binding of anAdoCbl to DDH induces Co$\mathrm{C}^{\prime}$ bond cleavage. The $\mathrm{Co}-\mathrm{C}^{\prime}$ bond cleavage is observed spectrophotometrically as the formation of cob(II)alamin either in the presence or absence of the substrate (28). However, the enzyme undergoes slow inactivation as observed by the formation of cob(III)alamin, irrespective of the presence of the substrate. Inactivation is a consequence of electron transfer from cob(II)alamin to the anhydroadenosyl radical and subsequent quenching of the allylic anion by a solvent derived proton (28).

Cleavage of the $\mathrm{Co}-\mathrm{C} 5$ ' bond of anAdoCbl generates the anhydroadenosyl radical and cob(II) alamin in the active site of DDH. The present paper presents EPR evidence for the presence of strongly-coupled spin-triplets involving the anhydroadenosyl radical and the low spin $\mathrm{Co}^{2+}$ of cob(II)alamin in complexes of DDH with the cofactor analog in the presence and absence of substrate. Analysis of the spin-triplet EPR spectra by simulation and interpretation of the ZFS in terms of molecular geometries is presented. 


\section{EXPERIMENTAL PROCEDURES}

\section{Materials}

Sodium cholate and AdoCbl were from Sigma; $(R, S)$-1,2-propanediol was from Aldrich; and $(R, S)-1,2-\left[{ }^{2} \mathrm{H}_{6}\right]$ propanediol $\left(99.5 \%{ }^{2} \mathrm{H}\right)$ was from CDN Isotopes. All other solvents, buffers and chemicals were obtained either from Fisher or Aldrich and used as supplied. anAdoCbl and $\left[{ }^{13} \mathrm{C}_{5}\right.$-ribosyl]-anAdoCbl were synthesized as described elsewhere (27). The ${ }^{13} \mathrm{C}$-labeled compound was prepared from the $\left[{ }^{13} \mathrm{C}_{5}\right.$-ribosyl]-anATP precursor, the synthesis of which is described elsewhere (29). Recombinant DDH from Salmonella typhimurium was produced in E. coli and purified and assayed as described previously $(28,30)$.

\section{Sample Preparation}

Samples were prepared from solutions that were kept within a Coy anaerobic chamber. The

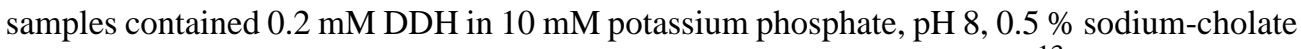
buffer in a final volume of $0.25 \mathrm{~mL}$, with either anAdoCbl $(0.32 \mathrm{mM})$ or $\left[{ }^{13} \mathrm{C}_{5}\right.$-ribosyl]anAdoCbl $(0.3 \mathrm{mM})$ as coenzyme. Samples prepared with substrate contained either $0.12 \mathrm{M}$ $(R, S)$-1,2-propandiol or $0.12 \mathrm{M}(R, S)$-1,2- $\left[{ }^{2} \mathrm{H}_{6}\right]$ propanediol. Substrate-free samples were preincubated for $20 \mathrm{~min}$ with $1 \mu \mathrm{M}$ AdoCbl before addition of coenzyme. This pretreatment with substoichiometric quantities of the normal cofactor ensured that residual traces of substrate, used to stabilize the enzyme during isolation and storage, would be converted to the product aldehyde. Samples were incubated at $25^{\circ} \mathrm{C}$ for $1-10 \mathrm{~min}$, transferred to EPR tubes and frozen by immersion of the tubes into cold isopentane $\left(-135^{\circ} \mathrm{C}\right)$.

\section{EPR Spectroscopy}

EPR spectra were obtained at cryogenic temperatures with a Varian spectrometer equipped with an E102 X-band microwave bridge, an Oxford Instruments ESR-900 continuous-flow helium cryostat, and an Oxford 3120 temperature controller. A Varian NMR gaussmeter, a Hewlett-Packard 5255A frequency converter and a 5245L electronic counter were used to measure the magnetic field strength and microwave frequency, respectively. The spectrometer was interfaced with a computer for data acquisition. Spin concentrations were estimated by double integration and comparison with a CuEDTA standard in water/glycerol.

\section{Spectral Simulations}

Fourier filtering methods $(31,32)$ were used to enhance resolution in the EPR spectra. The spectra were analyzed using the following spin Hamiltonian:

$$
H=\beta B \cdot g_{1} \cdot S_{1}+\beta B \cdot g_{2} \cdot S_{2}+S_{1} \cdot D \cdot S_{2}+J S_{1} \cdot S_{2}+H_{\text {nuc }}
$$

The first two terms in Equation 1 represent the Zeeman interaction of the low-spin $\mathrm{Co}^{2+}$ of cob (II)alamin and the anhydroadenosyl radical with the external magnetic field, respectively; the third and fourth terms represent the magnetic dipole-dipole ${ }^{2}$ (ZFS) and isotropic exchange interactions between cob(II)alamin and the anhydroadenosyl radical, respectively; and $\mathrm{H}_{n u c}$ represents the nuclear hyperfine interactions:

$$
H_{\mathrm{nuc}}=\sum_{i}\left(I_{i} \cdot A_{i 1} \cdot S_{1}\right)+\sum_{j}\left(I_{j} \cdot A_{j 2} \cdot S_{2}\right)
$$

In general, Euler rotations (33) are needed to bring each of the tensors in Equation 1 and Equation 2 into a common frame of reference, in this case the $g$-axis system of $\mathrm{Co}^{2+}$. However,

\footnotetext{
${ }^{2}$ The ZFS tensor used in the spin Hamiltonian has the form, $D=g_{1} \cdot D^{\prime} \cdot g_{2}$, where $D^{\prime}$ is the ZFS tensor expressed in the principal axis system of $g_{1}$ divided by the square of the free electron $g$-value. Using this formalism, contributions to the rhombicity of the ZFS from $g_{x y}$-anisotropy are considered directly and separately from the contributions due to electron spin delocalization.
} 
the $g$-tensors of organic radicals, such as the anhydroadenosyl radical, are very nearly isotropic and thus Euler angles are not required to align the two $g$-tensors. Also, the nuclear hyperfine interactions of the anhydroadenosyl radical and of cob(II)alamin, other than the ${ }^{59} \mathrm{Co}$-nucleus, are small relative to the ZFS and are safely neglected. The $g$-tensor and ${ }^{59} \mathrm{Co}$-hyperfine tensor of cob(II)alamin are collinear. Therefore, the Euler angles aligning the ZFS tensor with the $g$ tensor of cob(II)alamin (i.e., the angles relating the inter-spin vector to the $g$-axis system of $\mathrm{Co}^{2+}$ ) were the only angular variables in the fitting procedure. Energy levels and transition energies were obtained by diagonalization of the energy matrix. Field-swept powder EPR spectra were calculated as described previously (34). In this analysis, the ${ }^{59} \mathrm{Co}$-hyperfine interaction was treated to first-order, effectively reducing the energy matrix to a block diagonal form in the ${ }^{59} \mathrm{Co}$ nuclear quantum number, $m_{I}$. Initial estimates of the ZFS parameters were taken from the measured turning points in the experimental spectra, and the $\mathrm{Co}^{2+} \mathrm{g}$-values and hyperfine parameters were obtained from previously reported values of cob(II)alamin bound to methylmalonyl-CoA mutase (35). These values were refined (changed slightly) by trial-anderror until reasonable fits were obtained.

The triplet state spectra in the present study fall into the strongly exchange coupled regime, and in this regime $|J|$ does not influence the positions of transitions in the spectra (36). Likewise, simulations were not sensitive to the sign of $J$. Based on the observation that the signals were much weaker at $77 \mathrm{~K}$ than at $4 \mathrm{~K}$ and the implied (large ZFS) short inter-spin distances, the exchange coupling is likely ferromagnetic. An "operational" value of $|J|$ of $40-50 \mathrm{~cm}^{-1}$ was used in the simulations. Given that the $g$ tensor of the low-spin $\mathrm{Co}^{2+}$ is anisotropic and the inter-spin distances are short, there is a potential for anisotropic contributions from the exchange interaction $(36,37)$. Given the modest $\Delta g$ of low-spin $\mathrm{Co}^{2+}$, complications from delocalized unpaired spins are likely more significant than anisotropic exchange in this system. The anisotropy has been treated exclusively as arising from the dipole-dipole interaction.

\section{Analysis and Decomposition of the ZFS Tensor}

Due to the delocalization of spin within the anhydroadenosyl radical, the size of the $d_{z}^{2}$ orbital of $\mathrm{Co}^{2+}$, and the proximity of these two paramagnetic centers, the commonly invoked pointdipole approximation (relating the ZFS to the distance between the spins) is not adequate for this system. To overcome the limitations of the point-dipole approximation, and to exploit the structural information implicit in the ZFS tensor, a multipoint model known as the point-charge model was used (37-39). In this model, fractional spins are assigned to individual atoms or orbital lobes. The resulting ensemble of fractional spins is treated by summing the multiple dipole-dipole interactions occurring between the two paramagnetic centers in order to generate the ZFS tensor (Equation 3):

$D_{i j}=\frac{\mu_{o} \beta^{2}}{4 \pi} \sum_{m} \sum_{n} \rho_{1}(m) \rho_{2}(n) \frac{\left|r_{2}(n)-r_{1}(m)\right|^{2} \delta_{i j}-3\left(r_{2}(n)-r_{1}(m)\right)_{i}\left(r_{2}(n)-r_{1}(m)\right)_{j}}{\left|r_{2}(n)-r_{1}(m)\right|^{5}}$

In Equation 3, $\delta_{i j}$ is the Kronecker delta, $\rho_{1}(m)$ and $\rho_{2}(n)$ are the spin densities of the $m^{\text {th }}$ and $n^{\text {th }}$ fractional spin of $\operatorname{cob}(\mathrm{II})$ alamin and the anhydroadenosyl radical, respectively, and $\boldsymbol{r}_{\mathbf{1}}(\mathrm{m})$ and $\boldsymbol{r}_{\mathbf{2}}(n)$ are the corresponding position vectors (39). The relative position of the two paramagnetic centers was obtained by minimizing (with the aid of a simulated annealing algorithm) the sum of the squared differences between the experimental ZFS tensor elements and those calculated with equation $3(40,41)$. The search for reasonable local minima was guided by including in the objective function penalties to avoid unfavorable van der Waals contacts (42).

$$
F=F_{D}\left(1+f_{\text {vdw }}\right)
$$

In Equation $4, F_{D}$ has the form: 


$$
F_{D}=\sum_{i=1}^{2} \sum_{j=i}^{3}\left|D_{i j}^{\mathrm{exp}}-D_{i j}^{\mathrm{calc}}\right|^{2}
$$

where the sum includes the six independent ZFS tensor elements (defined by $D, E$ and the Euler angles $\zeta, \eta$, and $\xi) . f_{v d w}$ has the form:

$$
f_{\mathrm{vdw}}= \begin{cases}\sum_{i} \sum_{j}\left[\left(\frac{\sigma_{i j}}{r_{i j}}\right)^{12}-\left(\frac{\sigma_{i j}}{r_{i j}}\right)^{6}\right] & \text { for } \sigma_{i j}>r_{i j} \\ 0 & \text { for } \sigma_{i j}<r_{i j}\end{cases}
$$

where $\sigma_{i j}$ is the van der Waals collision diameter between the $i^{\text {th }}$ atom of the anhydroadenosyl radical and the $j^{\text {th }}$ atom of cob(II)alamin, and $r_{i j}$ is the corresponding distance.

The spin densities used in equation 3 (see Supporting Information) were obtained from singlepoint energy calculations on the anhydroadenosyl radical and a truncated cob(II)alamin structure using Gaussian 98 (43). The geometries of the paramagnetic centers were optimized using Becke-style 3-Parameter Density Functional Theory (DFT) with the Lee-Yang-Parr correlation functional (B3LYP) and Pople's polarized double- $\zeta$ 6-31G* basis set. Single-point calculations were performed on the optimized structures using the B3LYP hybrid functional in combination with the DFT-optimized valence triple- $\zeta$ basis set, TZVP. Isosurface plots of the spin densities of the anhydroadenosyl radical and the truncated cob(II)alamin are given in Figure 1A.

To better mimic the location of spin within the anhydroadenosyl radical, the spin density at each of the atoms in the $\pi$-system was divided in two and positioned at equal distances above and below the plane of the anhydroribosyl ring (38). The atoms in the $\pi$-system of the corrin ring of cob(II)alamin were treated in an analogous fashion. The spin of the corrin ring nitrogen atoms was split into an additional point corresponding to the $\sigma$-orbital coordinating the lowspin $\mathrm{Co}^{2+}$. The distribution of spin density within the $d_{z}^{2}$ orbital of $\mathrm{Co}^{2+}$, which can be expressed as a linear combination of $d_{\left(z-x^{2}\right)}{ }^{2}$ and $d_{\left(z^{2}-y^{2}\right)}$ orbitals, was approximated by dividing the spin into six points, two equidistant above and below the plane, and four equidistant within the plane of the corrin ring. The point-charge models of the anhydroadenosyl radical and cob(II)alamin that resulted in the best-fit ZFS tensors are illustrated in Figure 1B.

The starting coordinates for the analysis were obtained by placing the geometry optimized cob (II)alamin fragment and the adenine ring of the anhydroadenosyl radical in the positions observed in the crystal structure of DDH complexed with adeninylpentylcobalamin (44). The position of the anhydroribosyl moiety was varied by rotation about the glycosidic bond and small overall rotations and translations of the anhydroadenosyl radical were performed to find the best match of the experimentally observed and calculated ZFS tensors in the global minimization. The structure corresponding to the minimum in the analysis is not necessarily unique in its ability to reproduce the ZFS tensor. However, it represents the structure that best reproduces the ZFS tensor and while minimizing changes in the geometry from that expected from the crystallographic data (44).

\section{RESULTS AND DISCUSSION EPR with anAdoCbl as Coenzyme}

EPR spectra of DDH with anAdoCbl as coenzyme in the presence or absence of saturating 1,2propanediol are shown in Figure 2 and 3, respectively. These EPR spectra bear a resemblance to the strongly-coupled triplet spectra detected in the AdoCbl-dependent enzymes ribonucleotide reductase (45), glutamate mutase (46), and methylmalonyl-CoA mutase (47- 
49 ), in that the signals are centered at $g$-values midway between that of low-spin $\mathrm{Co}^{2+}$ and typical organic radicals and contain ${ }^{59} \mathrm{Co}$-hyperfine splittings that are contracted from that observed in magnetically isolated cob(II)alamin $(23,36)$. However, the width of the EPR spectra in Figure 2 and 3 are much greater than any of the aforementioned triplet spectra, indicating that the distance separating the two paramagnetic centers comprising the spin triplets in the present case is much smaller, resulting in a larger ZFS. The percentage of active sites hosting the triplet was determined from double integrations to be $\sim 70 \%$ in both cases. This value is consistent with the extent of $\mathrm{Co}-\mathrm{C}$ bond cleavage from similar samples estimated from $\mathrm{UV}-\mathrm{V}$ is spectroscopy after the same time period of incubation (28).

To establish the identity of the radical species spin-coupled to cob(II)alamin, EPR spectra were acquired with isotopically labeled coenzyme or substrate. Introduction of ${ }^{13} \mathrm{C}(I=1 / 2)$ at each position of the anhydroribosyl moiety of anAdoCbl ([ ${ }^{13} \mathrm{C}_{5}$-ribosyl $]$-anAdoCbl) leads to inhomogeneous broadening of the EPR signals both in the presence and absence of substrate (Figure $2 \mathrm{~B}$ and $3 \mathrm{~B}$, respectively). Only modest ${ }^{13} \mathrm{C}$-induced broadening effects are observed due to the large overall width of the EPR spectra and the hyperfine splitting contraction characteristic of strongly coupled triplet spin systems. The EPR spectrum obtained with unlabeled anAdoCbl and $(R, S)-1,2-\left[{ }^{2} \mathrm{H}_{6}\right]$ propanediol is given in Figure $2 \mathrm{C}$. A subtle line narrowing effect is observed due to incorporation of ${ }^{2} \mathrm{H}$ into the $5^{\prime}$-position of the coenzyme. Both these effects are more readily visible in the half-field $\left(\Delta \mathrm{M}_{S}=2\right)$ transitions wherein the intrinsic line widths are narrower (Figure 4 and Figure 5). The responses of the line widths to the magnetic isotope substitutions demonstrate that the anhydroadenosyl radical is the other half of the triplet spin system both in the presence and absence of substrate.

A weakly allowed half-field $\left(\Delta \mathrm{M}_{\mathrm{S}}=2\right)$ transition is a spectroscopic signature of stronglycoupled triplet spin systems $(35,50)$. Such transitions are observed in the EPR spectra of DDH with anAdoCbl, both in the presence and absence of substrate (Figure 4 and 5, respectively). Incubation of DDH with [ ${ }^{13} \mathrm{C}_{5}$-ribosyl]-anAdoCbl (Figure $4 \mathrm{~B}$ and $5 \mathrm{~B}$ ) or unlabeled anAdoCbl and $(R, S) 1,2-\left[{ }^{2} \mathrm{H}_{6}\right]$ propanediol (Figure 4C) cause line width effects in the half-field transitions analogous to those seen in the $\Delta \mathrm{M}_{\mathrm{S}}=1$ portion of the EPR spectra.

\section{Analysis of the EPR Spectra}

Spectral simulations were performed to better understand the complicated EPR spectra of DDH with anAdoCbl as coenzyme. The parameters used in the simulations are summarized in Table 1. Comparisons of the simulated and resolution enhanced experimental EPR spectra in the presence and absence of $(R, S)-1,2$-propanediol, are shown in Figure 6 and 7, respectively. The $g$-values used in the simulations were $g_{x}=2.30, g_{y}=2.21$, and $g_{z}=2.00$ for $\mathrm{Co}^{2+}$ and $g_{\text {iso }}=$ 2.00 for the anhydroadenosyl radical, which are typical for cob(II)alamin and organic radicals $(23,36,49)$. The only hyperfine interaction included in the spectral simulations was that derived from ${ }^{59} \mathrm{Co}\left(I=7 / 2 ; A_{x}=15 \mathrm{G}, A_{y}=5 \mathrm{G}, A_{z}=113 \mathrm{G}\right)$; all other hyperfine interactions contribute only to the line widths of the signals in EPR spectra.

The ZFS interactions between cob(II)alamin and the anhydroadenosyl radical are highly rhombic both in the presence $(D=-522 \mathrm{G}, \mathrm{E}=-91 \mathrm{G}, E / D=0.17)$ and absence $(D=-335 \mathrm{G}$, $\mathrm{E}=-87 \mathrm{G}, E / D=0.26$ ) of substrate, indicating that the electron spin density within the paramagnetic centers are highly delocalized, making the commonly invoked point dipole approximation invalid (39). Euler rotations were required to align the principal axis systems of the ZFS tensors with that of the $g$-tensor (or equivalently, the hyperfine splitting tensor) of $\mathrm{Co}^{2+}$, both in the presence $\left(\zeta=75^{\circ}, \eta=10^{\circ}, \xi=54^{\circ}\right)$ and absence $\left(\zeta=71^{\circ}, \eta=-10^{\circ}, \xi=\right.$ $90^{\circ}$ ) of substrate. This non-collinearity of tensor axes has a significant influence on the appearance of EPR spectra of strongly-coupled triplet systems in that it shifts the apparent $g$ values of turning points and transfers hyperfine splittings between turning points $(3,39)$. 
Figure 8 shows the geometries derived from the decomposition of the ZFS tensors obtained from the simulations of the EPR spectra of DDH with anAdoCbl in the presence (Figure 8A) and absence (Figure 8B) of (R,S)-1,2-propanediol, respectively. A comparison of these two structures reveals that upon substrate binding, there is only a slight perturbation in the position of the adenine ring (RMSD $0.53 \AA$ ). The position of the adenine ring of the anhydroadenosyl radical in both the presence and absence of substrate is very similar to that observed in the crystal structure of DDH complexed with adeninylpentylcobalamin (RMSD $0.67 \AA$ and 0.84 $\AA$, respectively ${ }^{3}$ ) (44). In addition, upon substrate binding, there is a rotation of the anhydroribosyl moiety by $\sim 60^{\circ}$ about the glycosidic bond, which postions the $5^{\prime}$-carbon of the anhydroadenosyl radical closer to $\mathrm{Co}^{2+}(3.5 \AA$ vs. $4.1 \AA)$. This structure is consistent with the larger ZFS observed in the EPR spectrum when substrate is present $(D=-522 \mathrm{G}$ vs. $D=-335$ $\mathrm{G})$. The basis for the greater rhombicity of the ZFS in the absence of substrate $(E / D=0.26$ vs. $E / D=0.17)$ is also evident from a comparison of the two structures. The vector between C-5' and $\mathrm{C}-3^{\prime}$ of the anhydroadenosyl radical (the two atoms with the greatest spin density in the radical) is very nearly perpendicular to the inter-spin vector with cob(II)alamin in the absence of substrate. In the substrate bound structure, the C-5'-C-3' vector and the inter-spin vector are closer to being parallel. In the case of electron delocalization about two points, the rhombicity in the ZFS is maximal when these two vectors are orthogonal and vanishes when they are collinear (39).

\section{Conclusions}

Rotation around the glycosidic bond of the anhydroadenosyl radical would position $\mathrm{C5}^{\prime}$ of the radical in close proximity to $\mathrm{C} 1$ of the substrate. Such a rotation mechanism for propagation of the radical was proposed earlier by Toraya and co-workers based on the crystal structure of the enzyme from $K$. oxytoca (44). The two positions of the anhydroadenosyl radical observed in the presence and absence of substrate in the current study provides direct evidence in support of this rotation mechanism.

The enzyme may be able to cleave the Co-C5' bond of AdoCbl without substrate present, but due to the instability of the $5^{\prime}$-deoxyadenosyl radical, the equilibrium for the bond homolysis reaction would favor the intact coenzyme. This scenario is supported by the results of this study, in which the equilibrium for Co-C5' bond homolysis of anAdoCbl is shifted towards formation of the cleavage products, due to the stability of the resulting allylic radical. Observation of the anhydroadenosyl radical supports the mechanism of DDH, where a transient 5 '-deoxyadenosyl radical intermediate functions as the direct hydrogen atom abstractor from substrate. In the case of the an $\mathrm{AdoCbl}$, the cofactor derived radical resides very close to its cob (II)alamin partner in the absence and presence of the substrate.

\section{Supplementary Material}

Refer to Web version on PubMed Central for supplementary material.

\section{REFERENCES}

1. Banerjee R. Radical peregrinations catalyzed by coenzyme $\mathrm{B}_{12}$-dependent enzymes. Biochemistry 2001;40:6191-6198. [PubMed: 11371179]

2. Marsh ENG, Drennan CL. Adenosylcobalamin-dependent isomerases: new insights into structure and mechanism. Curr. Opin. Chem. Biol 2001;5:499-505. [PubMed: 11578922]

3. Reed GH. Radical mechanisms in adenosylcobalamin-dependent enzymes. Curr. Opin. Chem. Biol 2004;8:477-483. [PubMed: 15450489]

\footnotetext{
${ }^{3}$ The orientation of the adenine ring has little effect on the calculated ZFS tensor. However, its position is constrained by the orientation of the glycosidic bond. The dihedral angle used to position the adenine ring was chosen so as to minimize the RMSD.
} 
4. Finke RG. Thermolysis of adenosylcobalamin: A product, kinetic, and Co-C5'sociation energy study. Inorg. Chem 1984;23:3041-3043.

5. Padmakumar R, Padmakumar R, Banerjee R. Evidence that cobalt-carbon bond homolysis is coupled to hydrogen atom abstraction from substrate in methylmalonyl-CoA mutase. Biochemistry 1997;36:3713-3718. [PubMed: 9132024]

6. Marsh ENG, Ballou DP. Coupling of cobalt-carbon bond homolysis and hydrogen atom abstraction in adenosylcobalamin-dependent glutamate mutase. Biochemistry 1998;37:11864-11872. [PubMed: 9718309]

7. Bandarian V, Reed GH. Isotope effects in the transient phases of the reaction catalyzed by ethanolamine ammonia-lyase: Determination of the number of exchangeable hydrogens in the enzyme-cofactor complex. Biochemistry 2000;39:12069-12075. [PubMed: 11009622]

8. Toraya $\mathrm{T}$. Radical catalysis in coenzyme $\mathrm{B}_{12}$-dependent isomerization (eliminating) reactions. Chem. Rev 2003;103:2095-2128. [PubMed: 12797825]

9. Booker S, Licht S, Broderick J, Stubbe J. Coenzyme B 12 -dependent ribonucleotide reductase: Evidence for the participation of five cysteine residues in ribonucleotide reduction. Biochemistry 1994;33:12676-12685. [PubMed: 7918494]

10. Chen D, Abend A, Stubbe J, Frey PA. Epimerization at carbon- $5^{\prime}-\left[5^{\prime}-2 \mathrm{H}\right]$ adenosylcobalamin by ribonucleoside triphosphate reductase: Cysteine 408-independent cleavage of the Co-C5' bond. Biochemistry 2003;42:4578-4584. [PubMed: 12693955]

11. Toraya, T. Diol dehydratase and glycerol dehydratase. In: Banerjee, editor. Chemistry and Biochemistry of $B_{12}$. NY: Wiley-Interscience; 1999. p. 783-809.

12. Walling C, Johnson RA. Fenton's reagent. Rearrangements during glycol oxidations. J. Am. Chem. Soc 1975;97:2405-2407.

13. Golding, BT.; Buckel, W. Corrin-dependent reactions. In: Sinnott, ML., editor. Comprehensive Biological Catalysis. London: Academic Press; 1997. p. 239-260.

14. Smith DM, Golding BT, Radom L. Understanding the mechanism of $\mathrm{B}_{12}$-dependent diol dehydratase: A synergistic retro-push-pull proposal. J. Am. Chem. Soc 2001;123:1664-1675. [PubMed: 11456766]

15. Kamachi T, Toraya T, Yoshizawa K. Catalytic roles of active-site amino acid residues of coenzyme $\mathrm{B}_{12}$-dependent diol dehyratase: protonation state of histidine and pull effect of glutamate. J. Am. Chem. Soc 2004;126:16207-16216. [PubMed: 15584757]

16. Shibata N, Masuda J, Tobimatsu T, Toraya T, Suto K, Morimoto Y, Yasuoka N. A new mode of $\mathrm{B}_{12}$ binding and the direct participation of a potassium ion in enzyme catalysis: $\mathrm{X}$-ray structure of diol dehydratase. Structure Fold. Des 1999:997-1008. [PubMed: 10467140]

17. Toraya T, Yoshizawa K, Eda M, Yamabe T. Direct participation of potassium ion in the catalysis of coenzyme B(12)-dependent diol dehydratase. J. Biochem. (Tokyo) 1999;126:650-654. [PubMed: 10502670]

18. Schwartz P, LoBrutto R, Reed GH, Frey PA. Suicide Inactivation of Dioldehydrase by 2Chloroacetaldehyde: Formation of the cis-ethanesemidione radical, and the role of a Monovalent Cation. Helv. Chim. Acta 2003;86:3764-3775.

19. Kawata M, Kinoshita K, Takahashi S, Ogura K, Komoto N, Yamanishi M, Tobimatsu T, Toraya T. Survey of catalytic residues and essential roles of glutamate- $\alpha 170$ and aspartate- $\alpha 335$ in coenzyme B 12 -dependent diol dehyratase. J. Biol. Chem 2006;281:18327-18334. [PubMed: 16571729]

20. Retey J, Umani-Ronchi A, Seibl J, Arigoni D. On the mechanism of the propanediol dehydrase reaction. Experientia 1966;22:502-503. [PubMed: 5968618]

21. Finlay TH, Valinsky J, Mildvan AS, Abeles RH. Electron spin resonance studies with dioldehydrase: evidence for radical intermediates in reactions catalyzed by coenzyme $\mathrm{B}_{12}$. J. Biol. Chem 1973;248:1285-1290. [PubMed: 4346950]

22. Valinsky JE, Abeles RH, Fee JA. Electron spin resonance studies on diol dehydrase III. Rapid kinetic studies on the rate of formation of radicals in the reaction with propanediol. J. Am. Chem. Soc 1974;96:4709-4710. [PubMed: 4368668]

23. Pilbrow, JR. EPR of $\mathrm{B}_{12}$-dependent enzyme reactions and related systems, in $\mathrm{B}_{12}$. In: Dolphin, D., editor. New York: John Wiley \& Sons, Inc; 1982. p. 431-463. 
24. Valinsky JE, Abeles RH, Mildvan AS. Electron spin resonance studies of diol dehydrase II. The hyperfine structure of proposed radical intermediates derived from isotopic substitution in 2chloroacetaldehyde. J. Biol. Chem 1974;249:2751-2755. [PubMed: 4364027]

25. Abend A, Bandarian V, Reed GH, Frey PA. Identification of cis-ethanesemidione as the organic radical derived from glycolaldehyde in the suicide inactivation of dioldehydrase and of ethanolamine ammonia-lyase. Biochemistry 2000;39:6250-6257. [PubMed: 10821701]

26. Yamanishi M, Hirofumi I, Murakami Y, Toraya T. Identification of the 1,2-propanediol-1-yl radical as an intermediate in adenosylcobalamin-dependent diol dehydratase reaction. Biochemistry 2005;44:2113-2118. [PubMed: 15697237]

27. Magnusson OT, Frey PA. Synthesis and characterization of $3^{\prime}, 4^{\prime}$-anhydroadenosylcobalamin: A coenzyme $B_{12}$ analogue with unusual properties. J. Am. Chem. Soc 2000;122:8807-8813.

28. Magnusson OT, Frey PA. Interactions of diol dehydrase and $3^{\prime}, 4^{\prime}$-anhydroadenosylcobalamin: Suicide inactivation by electron transfer. Biochemistry 2002;41:1695-1702. [PubMed: 11814365]

29. Magnusson OT, Reed GH, Frey PA. Characterization of an allylic analogue of the 5 '-deoxyadenosyl radical: An intermediate in the reaction of lysine 2,3-aminomutase. Biochemistry 2001;40:77737782. [PubMed: 11425303]

30. Poznanskaja AA, Tanizawa K, Soda K, Toraya T, Fukui S. Coenzyme B 12 -dependent diol dehydrase: purification, subunit heterogeneity, and reversible association. Arch. Biochem. Biophys 1979;194:379-386. [PubMed: 375836]

31. Kauppinen JK, Moffaatt DJ, Mantsch HH, Cameron DG. Fourier self-deconvolution: A method for resolving intrinsically overlapped bands. Appl. Spectrosc 1981;35:271-276.

32. Latwesen DG, Poe M, Leigh JS, Reed GH. Electron paramagnetic resonance studies of a ras p21MnIIGDP complex in solution. Biochemistry 1992;31:4946-4950. [PubMed: 1318075]

33. Goldstein, H.; Poole, CP., Jr; Safko, JL. Classical Mechanics. 3rd ed.. San Francisco: Addison Wesley; 2002.

34. Bandarian V, Reed GH. Hydrazine cation radical in the active site of ethanolamine ammonia-lyase: Mechanism-based inactivation by hydroxyethylhydrazine. Biochemistry 1999;38:12394-12402. [PubMed: 10493807]

35. Wertz, JE.; Bolton, JR. Electron Spin Resonance. New York: Chapman and Hall; 1986.

36. Gerfen, GJ. EPR spectroscopy of $\mathrm{B}_{12}$-dependent enzymes. In: Banerjee, R., editor. Chemistry and Biochemistry of $\mathrm{B}_{12}$. New York: Wiley-Interscience; 1999. p. 165-195.

37. Bencini, A.; Gatteschi, D. EPR of Exchange Coupled Systems. New York: Springer-Verlag; 1990.

38. Mukai K, Sogabe A. ESR studies of radical pairs of galvinoxyl radical in corresponding phenol matrix. J. Chem. Phys 1980;72:598-601.

39. Mansoorabadi, SO.; Reed, GH. Effects of electron spin delocalization and non-collinearity of interaction terms in EPR triplet powder patterns. In: Telser, J., editor. Paramagnetic resonance of metallobiomolecules. Washington, D. C.: American Chemical Society; 2003. p. 82-96.

40. Goffe WL, Ferrier GD, Rogers J. Global optimization of statistical functions with simulated annealing. J. Econometrics 1994;60:65-100.

41. Press, WH.; Flannery, BP.; Teukolsky, SA.; Vetterling, WT. Numerical Recipes. New York: Cambridge University Press; 1989.

42. Leach, AR. Molecular Modeling Principles and Applications. Second ed.. London: Prentice Hall; 2001.

43. Frisch, MJ.; Trucks, GW.; Schlegel, HB.; Scuseria, GE.; Robb, MA.; Cheeseman, JR.; Zakrzewski, VG.; Montgomery, JJA.; Stratmann, RE.; Burant, JC.; Dapprich, S.; Millam, JM.; Daniels, AD.; Kudin, KN.; Strain, MC.; Farkas, O.; Tomasi, J.; Barone, V.; Cossi, M.; Cammi, R.; Mennucci, B.; Pomelli, C.; Adamo, C.; Clifford, S.; Ochterski, J.; Petersson, GA.; Ayala, PY.; Cui, Q.; Morokuma, K.; Malick, DK.; Rabuck, AD.; Raghavachari, K.; Foresman, JB.; Cioslowski, J.; Ortiz, JV.; Baboul, AG.; Stefanov, BB.; Liu, G.; Liashenko, A.; Piskorz, P.; Komaromi, I.; Gomperts, R.; Martin, RL.; Fox, DJ.; Keith, T.; Al-Laham, MA.; Peng, CY.; Nanayakkara, A.; Gonzalez, C.; Challacombe, M.; Gill, PMW.; Johnson, B.; Chen, W.; Wong, MW.; Andres, JL.; Head-Gordon, M.; Replogle, ES.; Pople, JA. Gaussian 98. Pittsburg: Gaussian, Inc; 1998. 
44. Masuda J, Shibata N, Morimoto Y, Toraya T, Yasuoka N. How a protein generates a catalytic radical from coenzyme $\mathrm{B}_{12}$ : X-ray structure of a dioldehydratase-adeninylpentylcobalamin complex. Structure Fold Des 2000;8:775-788. [PubMed: 10903944]

45. Gerfen GJ, Licht S, Willems JP, Hoffman BM, Stubbe J. Electron paramagnetic resonance investigations of a kinetically competent intermediate formed in ribonucleotide reduction - evidence for thiyl radical-cob(II)alamin interaction. J. Am. Chem. Soc 1996;118:8192-8197.

46. Bothe H, Darley DJ, Albracht SP, Gerfen GJ, Golding BT, Buckel W. Identification of the 4-glutamyl radical as an intermediate in the carbon skeleton rearrangement catalyzed by coenzyme $\mathrm{B}_{12}$ dependent glutamate mutase from Clostridium cochlearium. Biochemistry 1998;37:4105-4113. [PubMed: 9521732]

47. Zhao Y, Abend A, Kunz M, Such P, Retey J. Electron paramagnetic resonance studies of the methylmalonyl-CoA mutase reaction. Evidence for radical intermediates using natural and artificial substrates as well as the competitive inhibitor 3-carboxypropyl-CoA. Eur. J. Biochem 1994;225:891896. [PubMed: 7957226]

48. Padmakumar R, Banerjee R. Evidence from electron paramagnetic resonance spectroscopy of the participation of radical intermediates in the reaction catalyzed by methylmalonyl-coenzyme $\mathrm{A}$ mutase. J. Biol. Chem 1995;270:9295-9300. [PubMed: 7721850]

49. Mansoorabadi SO, Padmakumar R, Fazliddinova N, Vlasie M, Banerjee R, Reed GH. Characterization of a succinyl-CoA radical-cob(II)alamin spin triplet intermediate in the reaction catalyzed by adenosylcobalamin-dependent methylmalonyl-CoA mutase. Biochemistry 2005;44:3153-3158. [PubMed: 15736925]

50. Eaton GR, Eaton SS. Resolved electron-electron spin-spin splittings in EPR spectra. Biol. Magn. Reson 1989;8:650. 
A

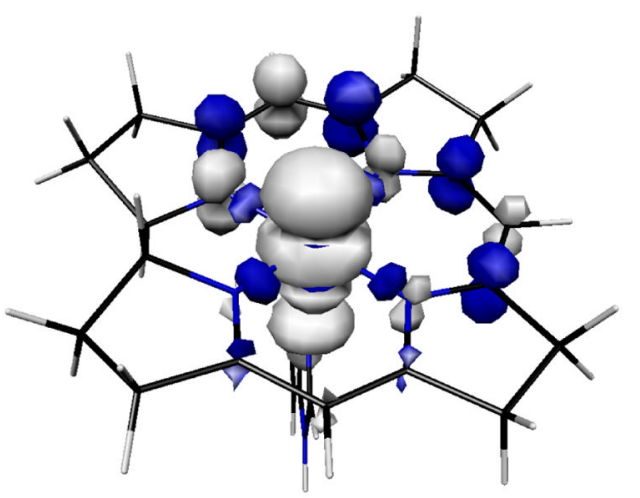

B

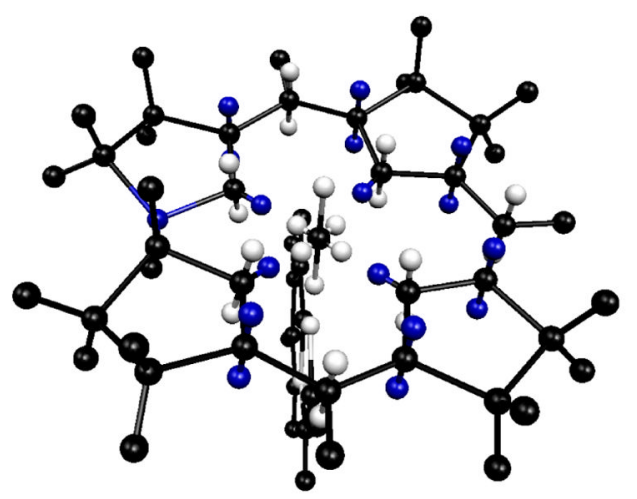

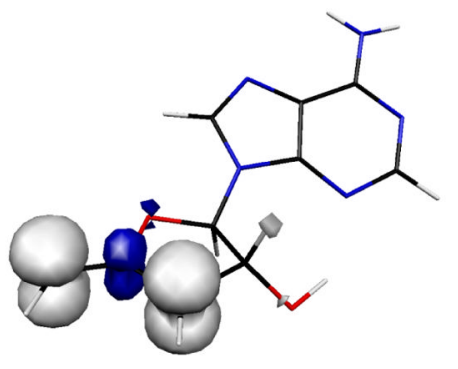

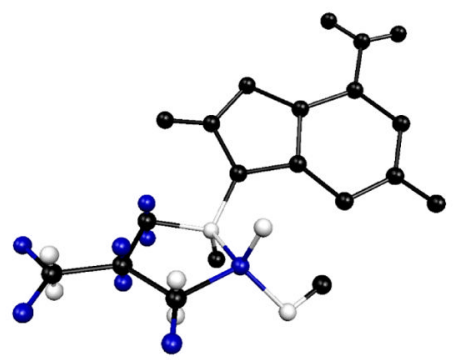

Figure 1.

Comparison of isosurface spin densities obtained from Gaussian 98 calculations A) and the point charge models used in the calculation of the ZFS tensor of the cob(II)alaminanhydroadenosyl radical.triplet system B). Positive spin density is in white, negative spin density in blue. In the point charge model, $\pi$-orbital lobes are positioned $0.7 \AA$ above and below the molecular planes (37), $\sigma$-orbital lobes of the equatorial ring nitrogens are $0.5 \AA$ from the nucleus along the Co-N bonds, and the $d_{\mathrm{z}}{ }^{2}$ orbital lobes are positioned $1.1 \AA$ above and below and $0.6 \AA$ within the plane of the corrin ring. 


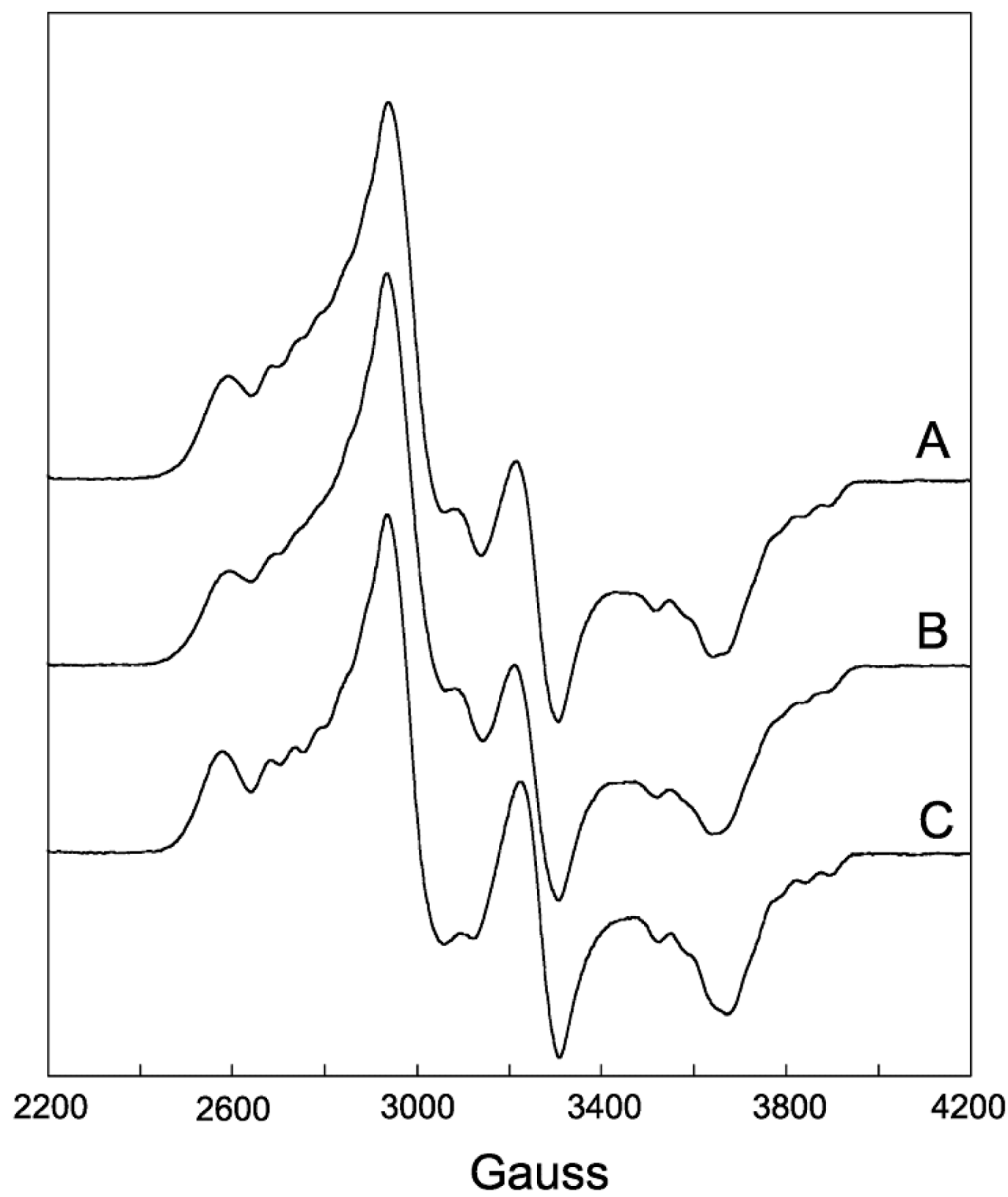

Figure 2.

EPR spectra of DDH with anAdoCbl as coenzyme in the presence of $(R, S)$-1,2-propanediol. The samples were frozen in cold isopentane $10 \mathrm{~min}$ after mixing within an anaerobic chamber. (A) Unlabeled coenzyme. (B) $\left[{ }^{13} \mathrm{C}_{5}\right.$-ribosyl]-anAdoCbl. (C) anAdoCbl and $(R, S)-1,2-\left[{ }^{2} \mathrm{H}_{6}\right]$ propanediol. Deuterium labeling at $\mathrm{C}^{\prime}$ ' of the coenzyme occurs upon reaction with substrate. Experimental conditions: temperature, $4.5 \mathrm{~K}$; modulation amplitude, $12 \mathrm{G}$; microwave power $0.2 \mathrm{~mW}$; spectrometer frequency, $9.233 \mathrm{GHz}(g=2.0$ at 3,299 G). All spectra are an average of four 8 min scans. 


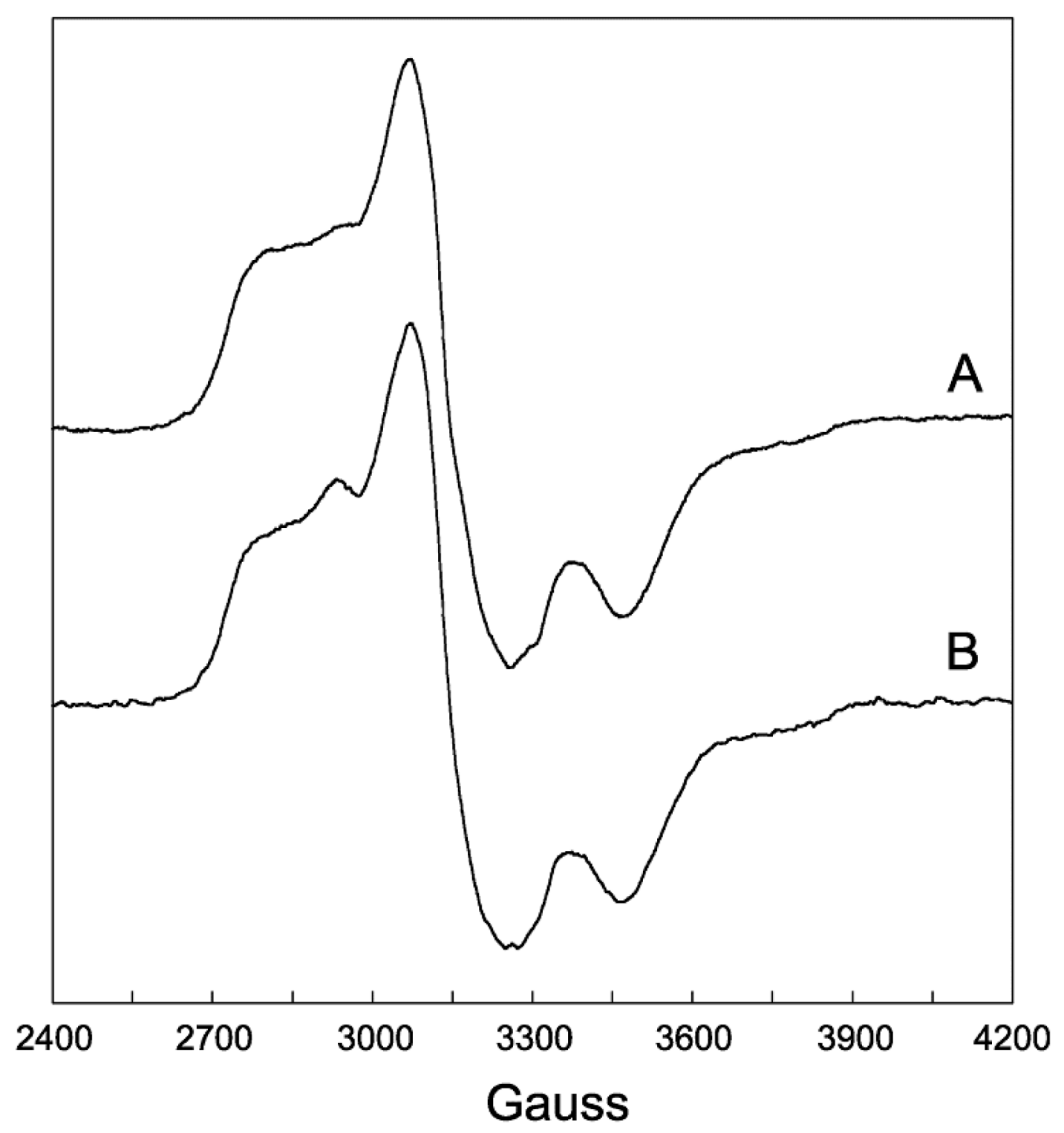

Figure 3.

EPR spectra of DDH with anAdoCbl as coenzyme in the absence of substrate. The samples were frozen in cold isopentane $\sim 1$ min after mixing within an anaerobic chamber. (A) Unlabeled coenzyme. (B) $\left[{ }^{13} \mathrm{C}_{5}\right.$-ribosyl]-anAdoCbl. Experimental conditions: temperature, $4.5 \mathrm{~K}$; modulation amplitude, $12 \mathrm{G}$; microwave power $0.2 \mathrm{~mW}$; spectrometer frequency, 9.233 $\mathrm{GHz}$. Both spectra are an average of four 8 min scans. 


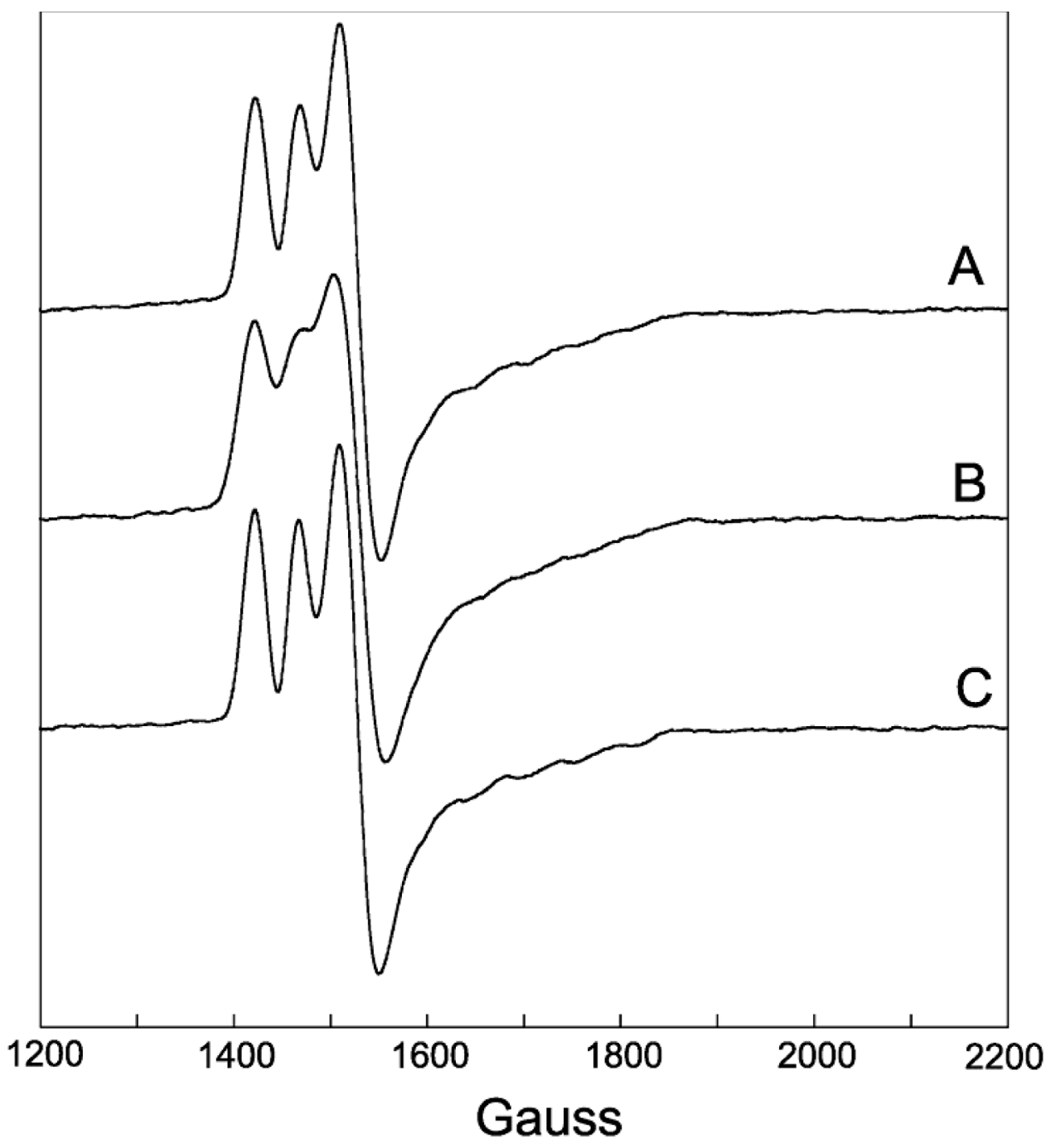

Figure 4.

EPR spectra of the half-field $\left(\Delta \mathrm{M}_{\mathrm{S}}=2\right)$ transition for the same samples as described in Figure 2. Experimental conditions: temperature, $4.5 \mathrm{~K}$; modulation amplitude, $12 \mathrm{G}$; microwave power $2 \mathrm{~mW}$; spectrometer frequency, $9.233 \mathrm{GHz}$. All spectra are an average of four $8 \mathrm{~min}$ scans. 


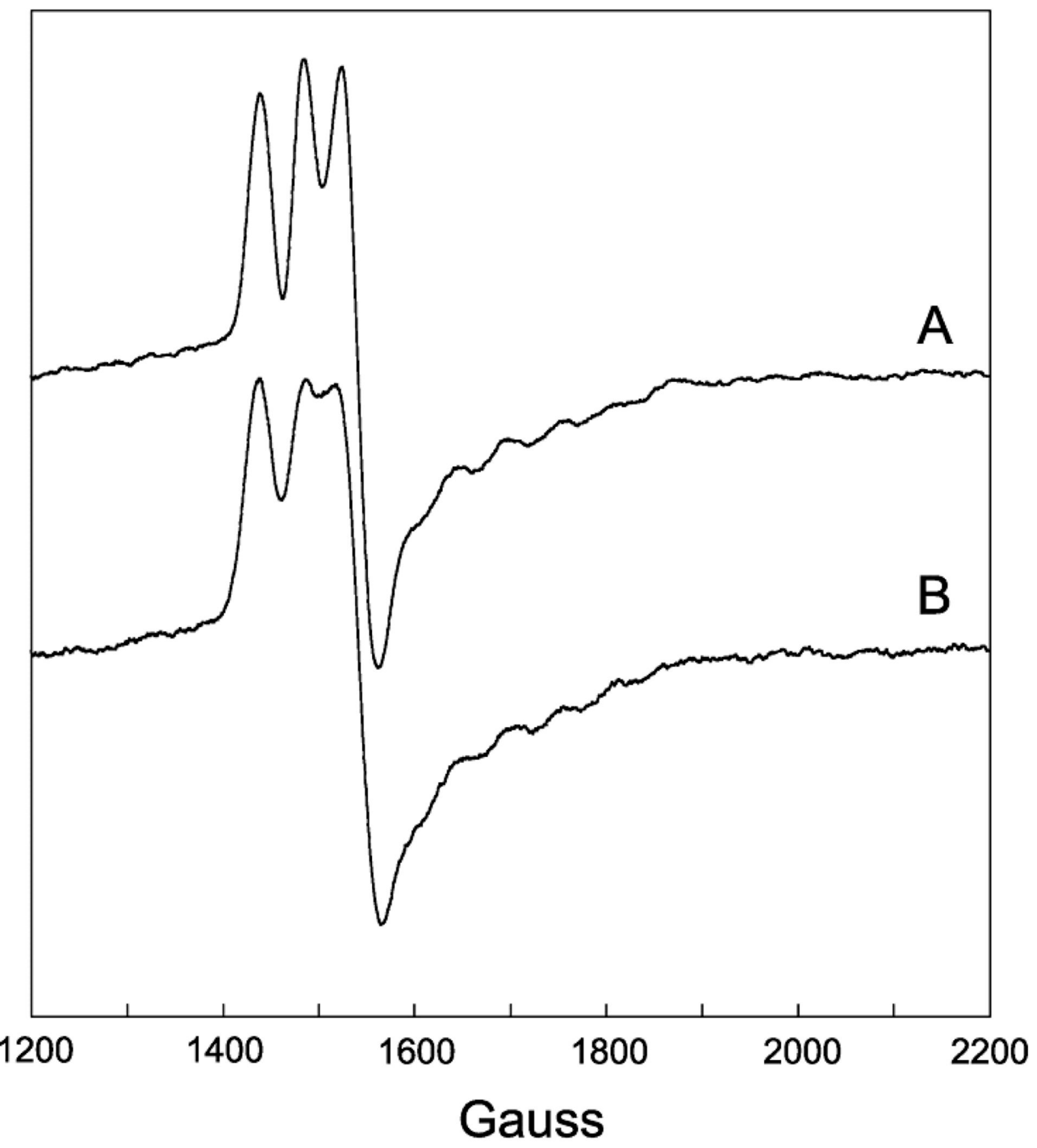

Figure 5.

EPR spectra of a half-field $\left(\Delta \mathrm{M}_{\mathrm{S}}=2\right)$ transition for the same samples as described in Figure 3. Experimental conditions: temperature, $4.5 \mathrm{~K}$; modulation amplitude, $12 \mathrm{G}$; microwave power $2 \mathrm{~mW}$; spectrometer frequency, $9.233 \mathrm{GHz}$. All spectra are an average of four $8 \mathrm{~min}$ scans. 


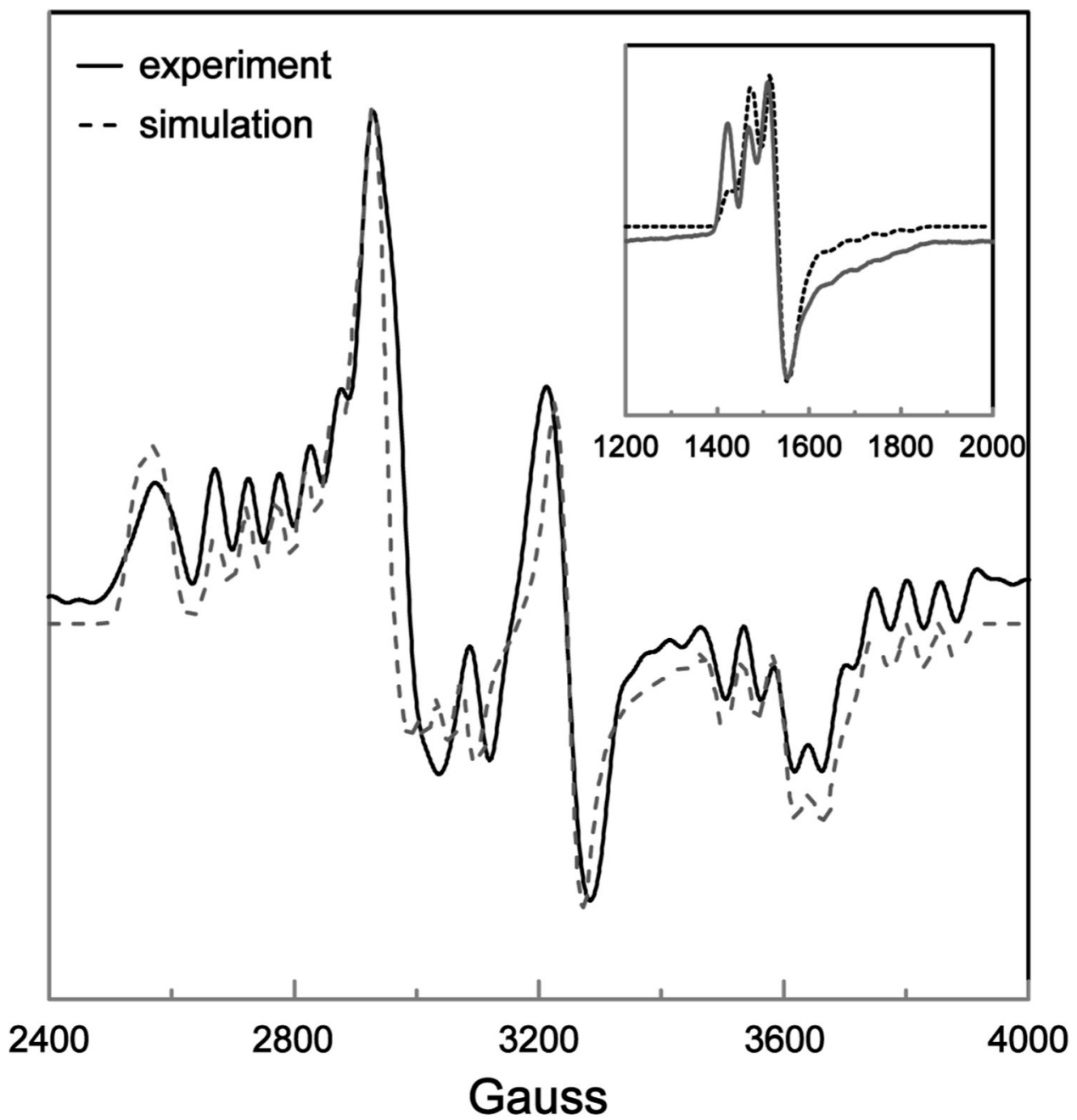

Figure 6.

Comparison of the simulated and resolution enhanced experimental EPR spectrum of DDH with anAdoCbl in the presence of saturating $(R, S)$-1,2-propanediol. The ZFS parameters were $D=-522 \mathrm{G}$ and $E=-91 \mathrm{G}$, with Euler angles $\zeta=75^{\circ}, \eta=10^{\circ}$, and $\xi=54^{\circ}$. The $g$-values for cob(II)alamin and the anhydroadenosyl radical $g_{x}=2.30, g_{y}=2.21, g_{z}=2.00$ and $g_{\text {iso }}=2.00$, respectively. The ${ }^{59} \mathrm{Co}$-hyperfine splittings were $A_{x}=15 \mathrm{G}, A_{y}=5 \mathrm{G}$ and $A_{z}=113 \mathrm{G}$. Lorentzian line shapes were used with isotropic line widths of $15 \mathrm{G}$. The inset compares the simulated and experimental EPR spectrum of the half-field transition, obtained with the same parameter set. 


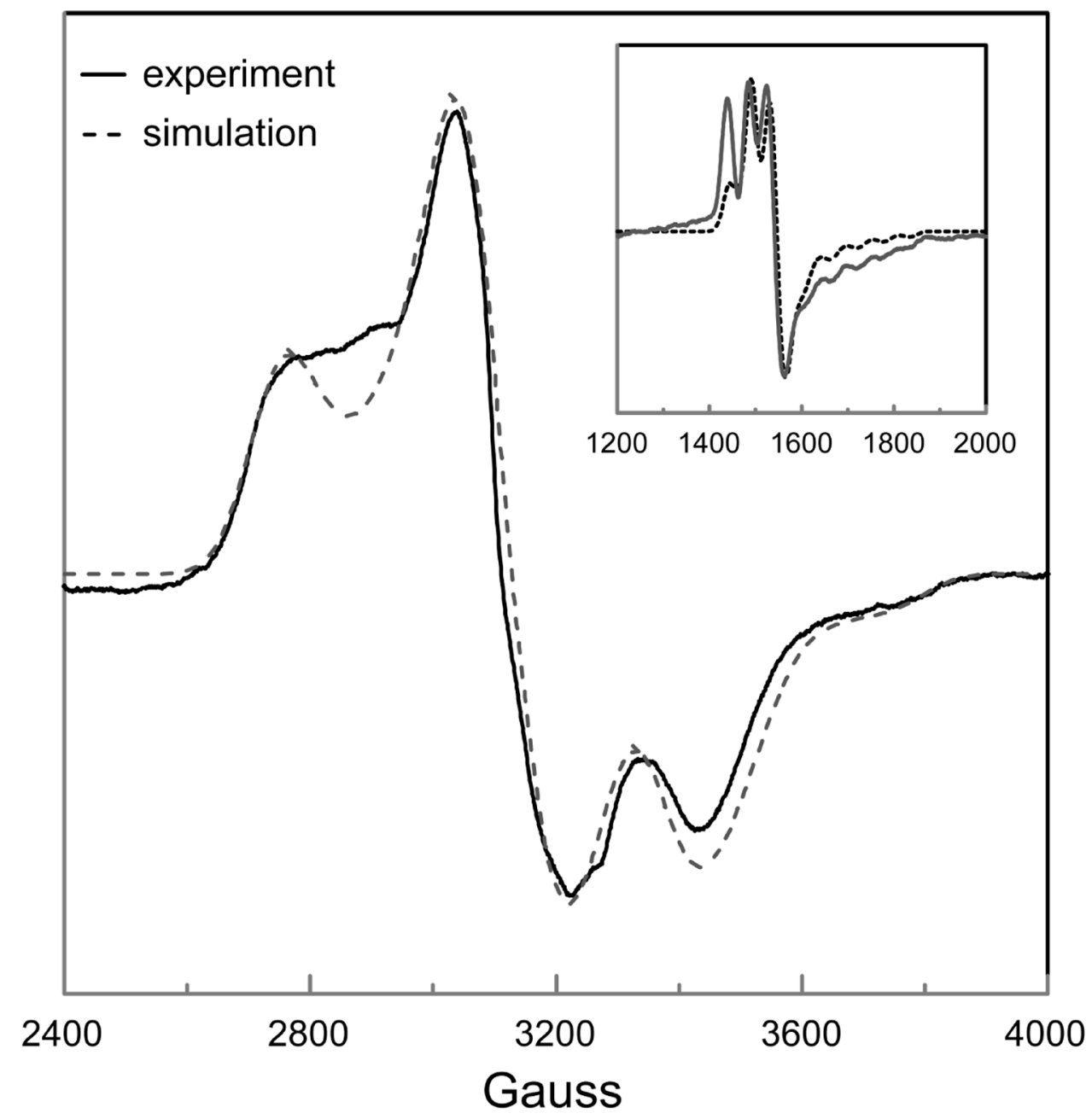

Figure 7.

Comparison of the simulated and experimental EPR spectrum of DDH with anAdoCbl in the absence of $(R, S)-1,2$-propanediol. The ZFS parameters were $D=-335 \mathrm{G}$ and $E=-87 \mathrm{G}$, with Euler angles $\zeta=71^{\circ}, \eta=-10^{\circ}$, and $\xi=90^{\circ}$. The $g$-values for cob(II)alamin and the anhydroadenosyl radical $g_{x}=2.30, g_{y}=2.21, g_{z}=2.00$ and $g_{i s o}=2.00$, respectively. The ${ }^{59}$ Co-hyperfine splittings were $A_{x}=15 \mathrm{G}, A_{y}=5 \mathrm{G}$ and $A_{z}=113 \mathrm{G}$. Lorentzian line shapes were used with isotropic line widths of $55 \mathrm{G}$. The inset compares the simulated and experimental EPR spectrum of the half-field transition, obtained with the same parameter set. 


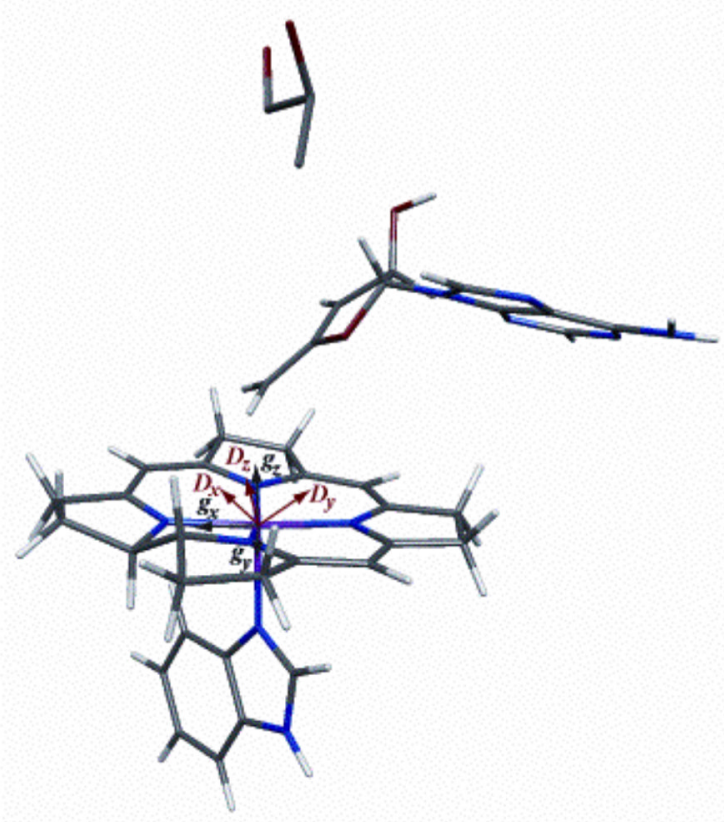

A

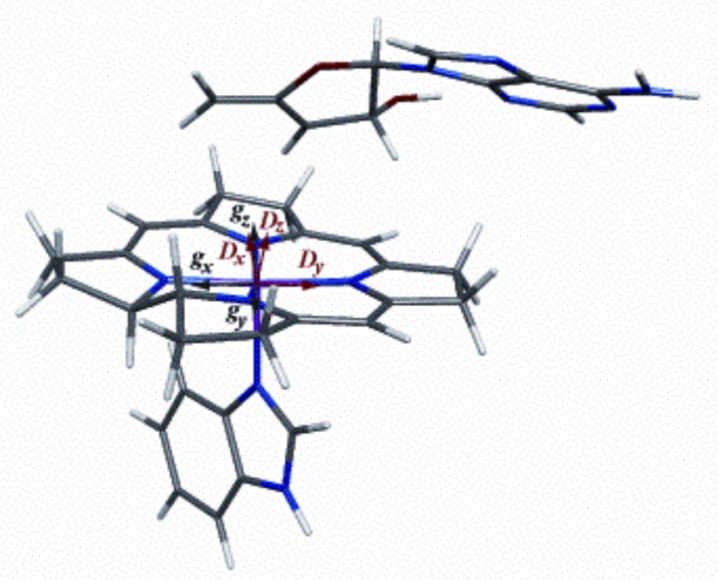

B

Figure 8.

Model of cob(II)alamin and the anhydroadenosyl radical in the active site of DDH in the presence A) and absence B) of ( $R, S)$-1,2-propanediol. A) The distance between $\mathrm{C} 5^{\prime}$ and $\mathrm{Co}^{2+}$ is $3.5 \AA$. The position of the adenine ring is very similar to that observed in the crystal structure of the DDH- adeninylpentylcobalamin complex (RMSD, $0.67 \AA$ ) (44). B) The distance between $\mathrm{C}^{\prime}$ and $\mathrm{Co}^{2+}$ is $4.1 \AA$. The position of the adenine ring is perturbed slightly (RMSD, $0.53 \AA$ ) and the anhydroribosyl moiety rotates $\sim 60^{\circ}$ relative to their positions when substrate is bound. The positions of the $g$ and $\boldsymbol{D}$ axes are labeled in the corin ring. 


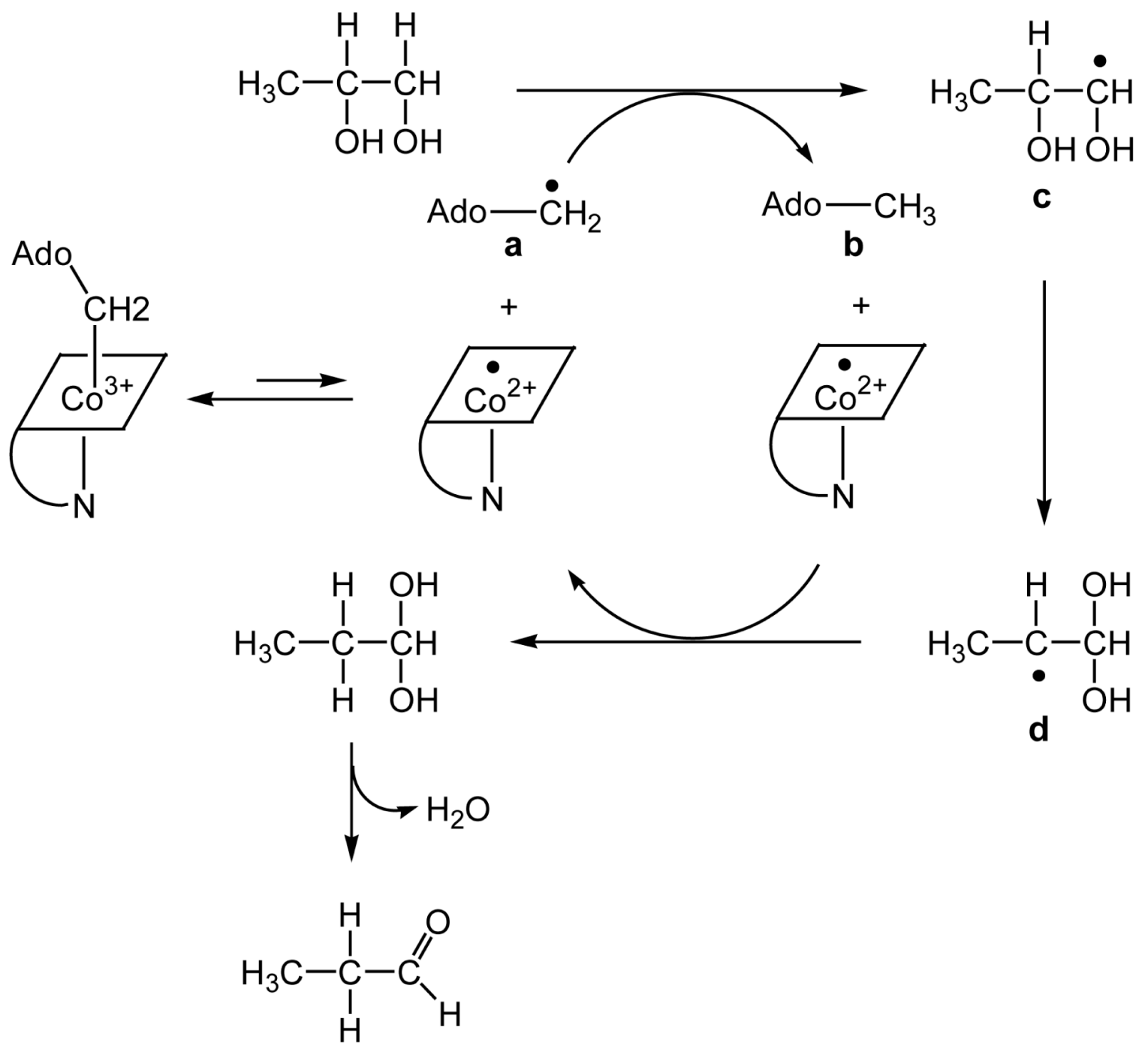

Scheme 1. 


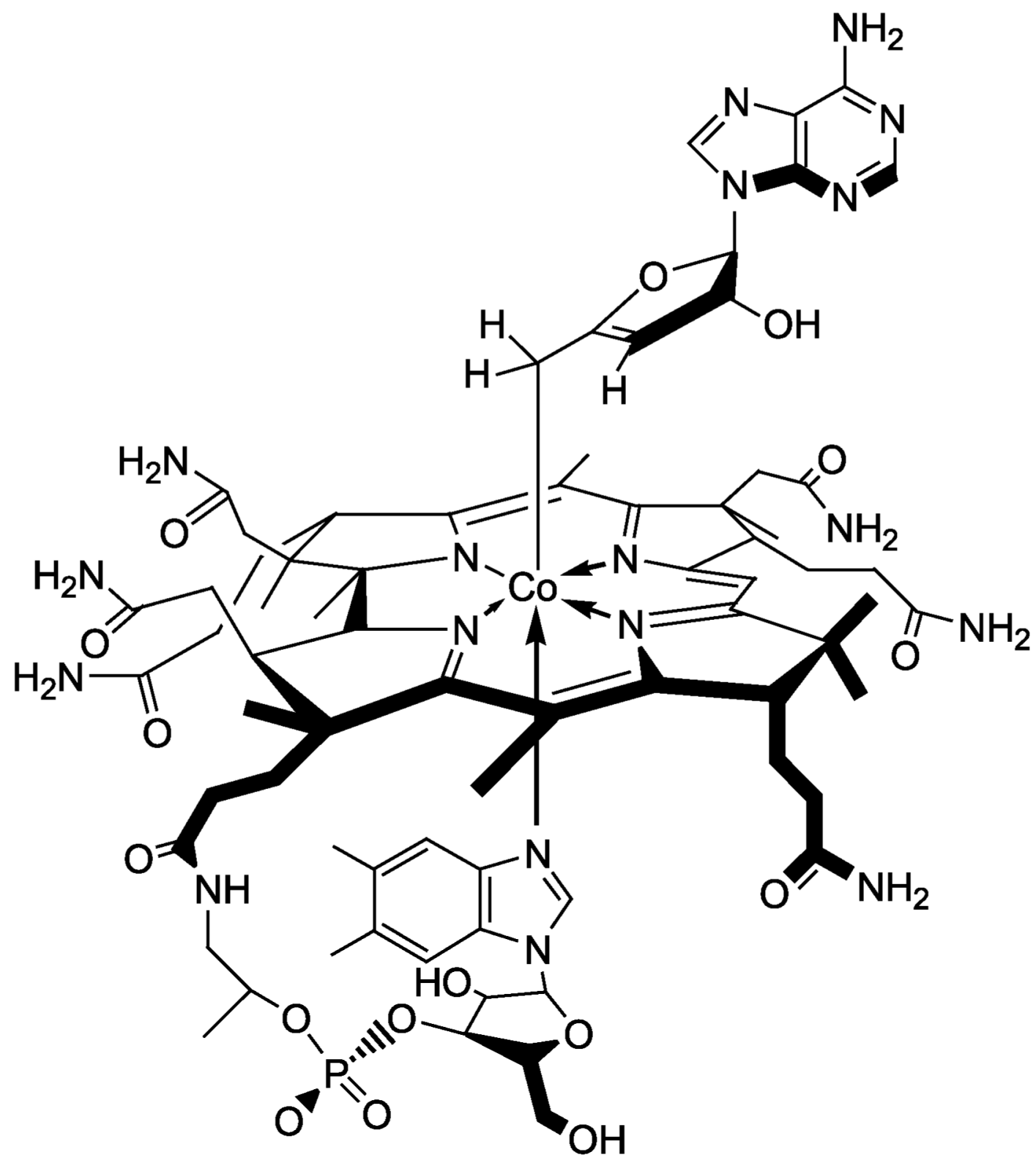

Scheme 2. 

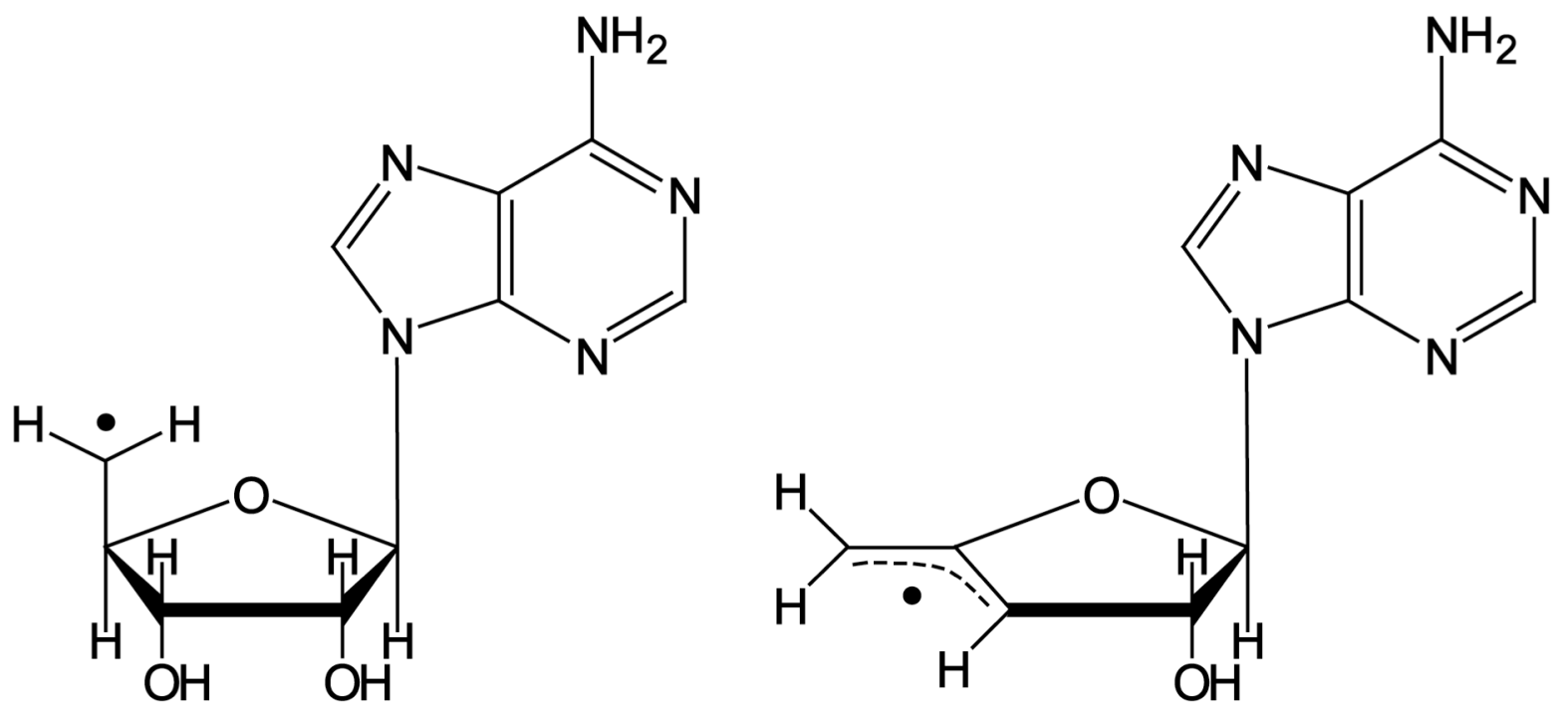

Scheme 3. 
Table 1

Parameters used in the simulation of the EPR spectra of DDH with anAdoCbl in the presence and absence of $(R, S)-1,2-$ propanediol.

\begin{tabular}{|c|c|c|}
\hline (R,S)-1,2-propanediol & Present & Absent \\
\hline $\begin{array}{c}\mathrm{Co}^{2+} g \text {-values } \\
\text { Radical } g \text {-values } \\
{ }^{59} \text { Co-hyperfine tensor } \\
\text { ZFS parameters } \\
\text { Euler angles } \\
\text { Exchange coupling }\end{array}$ & $\begin{array}{c}g_{x}=2.30, g_{y}=2.21, g_{z}=2.00 \\
g_{\text {iso }}=2.00 \\
A_{x}=15 \mathrm{G}, A_{y}=5 \mathrm{G}, A_{z}=113 \mathrm{G} \\
D=-522 \mathrm{G}, E=-91 \mathrm{G} \\
\zeta=75^{\circ}, \eta=10^{\circ}, \xi=54^{\circ} \\
|J| \sim 40-50 \mathrm{~cm}^{-1}\end{array}$ & $\begin{array}{c}g_{x}=2.30, g_{y}=2.21, g_{z}=2.00 \\
g_{\text {iso }}=2.00 \\
A_{x}=15 \mathrm{G}, A_{y}=5 \mathrm{G}, A_{z}=113 \mathrm{G} \\
D=-335 \mathrm{G}, E=-87 \mathrm{G} \\
\zeta=71^{\circ}, \eta=-10^{\circ}, \xi=90^{\circ} \\
|J| \sim 40-50 \mathrm{~cm}^{-1}\end{array}$ \\
\hline
\end{tabular}

